\title{
Spectral Properties of Circular Piezoelectric Unimorphs
}

\author{
Martin PUSTKA(1), Ladislav PƯST ${ }^{(2)}$ \\ (1) VÚTS, a.s. \\ Svárovská 619, 46001 Liberec XI, Czech Republic; e-mail: martin.pustka@vuts.cz \\ (2) Institute of Thermomechanics $A S$ CR, v.v.i. \\ Dolejškova 5, 18200 Praha 8, Czech Republic
}

(received June 29, 2016; accepted May 8, 2017)

\begin{abstract}
The piezoelectric unimorphs are essential resonant components of many oscillating systems including electroacoustic devices. The unimorph spectral properties are namely dependent on geometric dimensions, applied materials and mounting. Preliminary dimensioning and optimization of unimorph shape are usually carried out prior to comprehensive design work mostly based on finite element method. Simple analytical model is a suitable tool for initial design phase. This paper presents a derivation of calculation model describing natural vibrations of a circular unimorph with the piezoelectric layer diameter smaller than the elastic layer diameter. The system of equations with closed-form solution is instrumental to calculation of resonant frequencies and mode shapes for unimorphs with clamped, simply supported and free circumference. The theoretical results are compared with vibration velocity measurement of clamped unimorph sample in a wide frequency range. Analytical model derived in this paper is used to assess the effect of the thickness tolerance on unimorph resonant frequencies.
\end{abstract}

Keywords: piezoelectric unimorph; natural vibrations; resonant frequency; mode shape.

\section{Introduction}

Piezoelectric composite plates operating in bending mode are widely used in many applications as electroacoustic transducers, actuating and sensing elements or energy harvesting components. The structure of a piezoelectric composite plate is relatively simple and has two basic designs. The unimorph element consists of one piezoelectric layer and one elastic layer (commonly metallic) bonded together. The bimorph element is composed of two piezoelectric layers, alternatively with a central elastic layer, usually possessing a symmetry about the middle plane. The application of an electric field across the electrodes produces lateral expansion of piezoelectric layer(s) resulting in a bending deformation of the whole structure. Equally, flexural excitation of a composite plate results in the generation of electrical charge on the electrodes.

The design process of devices utilizing piezoelectric plates involves dimensioning and performance optimization of the key elements. For dynamic applications with resonant structures the information about the plate spectral properties (natural frequencies and mode shapes) is also of importance. The initial design and sensitivity analysis using finite element method are influenced by mesh modification needs associated with time costs. Therefore, suitable analytical models with closed-form solution are essential and highly conducive.

Theoretical analyses of piezoelectric composite plates are mostly based on Kirchhoff's laminated plate theory. Previous analytical works were usually focused mainly on static deflection solution under various types of loading. A dynamic solution was studied seldom. Derived expressions are valid from static regime to the vicinity of fundamental resonance frequency. Simply supported unimorph with piezoelectric layer of the same diameter as the elastic layer was studied in (Adelman, Stavsky, 1980) for circular shape and in (LI et al., 1999) for rectangular and circular shapes. The analysis of a simply supported unimorph structure composed of elastic, piezoelectric and bonding layer with the diameter of the piezoelectric layer smaller than that of the elastic layer was presented in (LI, Chen, 2003). The model of a clamped multilayer unimorph where the diameter of the elastic layer is greater 
than that of the remaining layers was introduced in (Deshrande, Saggere, 2007). Analogical analyses considering various unimorph geometry and boundary conditions at the edges were provided, e.g. in (Mo et al., 2006; Dong et al., 2007) or (Mo et al., 2010).

Analytical model for the circular bimorph in the form of layered shell of revolution was presented in (Dobrucki, Pruchnicki, 1997). The equations were solved numerically by means of finite element analysis and verified experimentally for a bimorph clamped at the center and free on the circumference. PRASAD et al. (2006) derived an analytical lumped-element model for a clamped circular unimorph based on classical laminated plate theory. Similar approach was used in (PAPILA et al., 2008) for the static solution of clamped actuators in a configuration of an inner piezoelectric disc and an outer piezoelectric ring connected to an elastic layer. YANG et al. (2006) used two-dimensional equations of higher-order plate theory for a solution of static deflection of simply supported unimorph with the piezoelectric layer of the same radius as an elastic layer.

Only few papers were devoted to a problem of natural vibration and spectral properties. The bendingmode resonant frequencies for simply supported circular and rectangular unimorphs with piezoelectric covering the entire elastic layer were introduced in (LI et al., 1999). The approximate analytical method for evaluating fundamental vibration mode parameters of clamped unimorph with the diameter of the piezoelectric layer smaller than that of the elastic layer are treated in (LIU et al., 2003). Overtone vibration modes were evaluated using FEM. The free vibration analysis of circular bimorph plate under various boundary conditions was presented in (WANG et al., 2001), where all bimorph layers are of the same diameter. Dynamic properties were mentioned only as additional remarks to the static solutions without any detailed analysis in the above mentioned publications. The current manuscript fulfils this lack and presents a detailed analytical solution of frequency and modal spectra of the studied system.

This paper provides an analytical study of natural vibration for circular unimorph with the piezoelectric layer diameter smaller than the elastic layer diameter (Fig. 1). The basic relations are derived using Kirchhoff's theory for bending of thin plates (LEISSA, 1969) in polar coordinates. As the accuracy of computational models is generally limited by non-linear properties of piezoelectric ceramics and a high variability of its material constants, both layers are supposed to be linearly isotropic for simplicity. Regarding the complete axial symmetry of the structure and symmetric excitation the vibrations are assumed to be also axially symmetric with nodal circles but without nodal diameters. In a polarized accurately shaped piezoceramic disc only modes having symmetry with respect to the

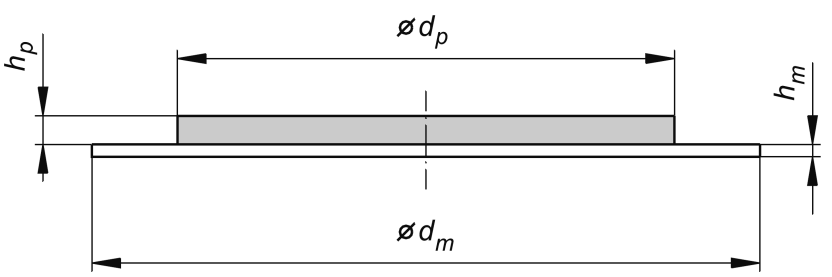

Fig. 1. Circular piezoelectric unimorph cross section.

disc axis can be excited by application of an electric field (GAzIs, Mindlin, 1960). The final closed-form solution comprises various boundary conditions on the unimorph outer circumference (clamped, simply supported and free).

\section{Computational model}

The piezoelectric unimorph structure composed of two axisymmetric circular plates is depicted in Fig. 1. For a calculation we assume that both plates are isotropic and bonding between plates is absolute. The bonding layer thickness as well as thickness of metallic electrodes is one order of magnitude smaller than the thickness of piezoelectric and elastic elements and can be neglected in the current model. The influence of the bonding layer on the model parameters is shown in Table 1 . The structure is solved as two plates connected on diameter $d_{p}$, central solid of thickness $h_{1}=h_{p}+h_{m}$ and outer annular of thickness $h_{2}=h_{m}$. The equivalent computational model of piezoelectric unimorph is shown in Fig. 2, where the solid plate is considered homogenous with equivalent parameters and the neutral surface is common for both plates.

Table 1. Influence of bonding layer thickness on the values of equivalent Young's modulus $E_{1}$ and neutral plane position $c$.

\begin{tabular}{|c|c|c|c|c|c|c|}
\hline$h_{a}[\mu \mathrm{m}]$ & 0 & 2 & 4 & 6 & 8 & 10 \\
\hline$E_{1}[\mathrm{GPa}]$ & 87.897 & 87.559 & 87.229 & 86.907 & 86.591 & 86.283 \\
\hline$E_{1} / E_{10}$ & 1 & 0.9962 & 0.9924 & 0.9887 & 0.9851 & 0.9816 \\
\hline$c[\mu \mathrm{m}]$ & 129.7 & 131.1 & 132.6 & 134.0 & 135.5 & 136.9 \\
\hline
\end{tabular}

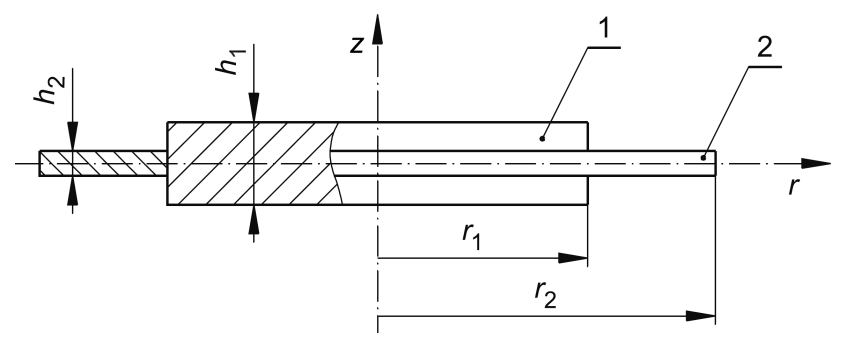

Fig. 2. The equivalent computational model of piezoelectric unimorph composed of central solid plate and outer annular plate. 


\subsection{Fundamental relations for thin plate vibration in polar coordinates}

The equation of motion for a transverse displacement $w$ in the $z$ direction of a thin plate has a form (LEISSA, 1969)

$$
\rho^{*} \frac{\partial^{2} w}{\partial t^{2}}+D \nabla^{4} w=0
$$

where $\nabla^{4}=\nabla^{2} \nabla^{2}$ and

$$
\nabla^{2}=\frac{\partial^{2}}{\partial r^{2}}+\frac{1}{r} \frac{\partial}{\partial r}+\frac{1}{r^{2}} \frac{\partial^{2}}{\partial \varphi^{2}}
$$

is the Laplacian operator expressed in polar coordinates $r, \varphi$,

$$
D=\frac{E h_{0}^{3}}{12\left(1-\mu^{2}\right)}
$$

is plate flexural rigidity, $E$ is Young's modulus, $h_{0}-$ plate thickness, $\mu$ - Poisson's ratio, $\rho^{*}$ - plate mass per unit area, and $t$ is time.

We suppose the solution of natural vibrations in the form of a harmonic wave

$$
w(r, \varphi, t)=W(r, \varphi) \sin (\Omega t),
$$

where $\Omega$ is angular frequency. Substituting this formula into equation of motion (1) we arrive at a simpler relation for two variables $r, \varphi$ only

$$
-\lambda^{4} W(r, \varphi)+\nabla^{4} W(r, \varphi)=0,
$$

where the frequency parameter is

$\lambda^{4}=\frac{\Omega^{2} \rho^{*}}{D}=\frac{12 \Omega^{2} \rho^{*}\left(1-\mu^{2}\right)}{E h_{o}^{3}}=\frac{12 \Omega^{2} \rho\left(1-\mu^{2}\right)}{E h_{o}^{2}}$,

and $\rho=\rho^{*} / h_{0}$ is density. Equation (5) can be split into two parts

$$
\begin{aligned}
& \nabla^{2} V_{1}+\lambda^{2} V_{1}=0, \\
& \nabla^{2} V_{2}-\lambda^{2} V_{2}=0 .
\end{aligned}
$$

The general solution of Eqs. (7) is given by

$V_{i}(r, \varphi)=\left(A_{n} \cos n \varphi+B_{n} \sin n \varphi\right) V_{i n}(r), \quad i=1,2$,

where $A_{n}, B_{n}$ are constant amplitudes. Number $n$ is connected with the number of nodal diameters and/or with the deformation of umbrella mode corresponding to $n=0$. The azimuthal imperfection can trigger $n \neq 0$ modes, but these are very small compared to the zero mode in carefully made and mounted unimorphs. We suppose that the flexural deformation of the unimorph is excited by axisymmetric radial vibration of piezoelectric layer and the solution has nodal circles only. Then $n=0$ in Eq. (8), the term in parentheses is constant and Eqs. (7) have a form (LEISSA, 1969)

$$
\begin{aligned}
& \left(\frac{\partial^{2}}{\partial r^{2}}+\frac{1}{r} \frac{\partial}{\partial r}\right) V_{1}+\lambda^{2} V_{1}=0 \\
& \left(\frac{\partial^{2}}{\partial r^{2}}+\frac{1}{r} \frac{\partial}{\partial r}\right) V_{2}-\lambda^{2} V_{2}=0,
\end{aligned}
$$

with a final solution

$$
\begin{aligned}
& V_{1}=A J_{0}(\lambda r)+B Y_{0}(\lambda r), \\
& V_{2}=C I_{0}(\lambda r)+D^{*} K_{0}(\lambda r),
\end{aligned}
$$

where $J_{0}, Y_{0}$ are Bessel's functions of the first and second kind and zero-th order, $I_{0}, K_{0}$ are modified Bessel's functions of the first and second kind and zeroth order and $A, B, C, D^{*}$ are constant amplitudes. Functions $K_{0}(\lambda r)$ and $Y_{0}(\lambda r)$ are infinite at the origin $r=0$ and therefore $B=D^{*}=0$ for a solid plate.

Plate mounting on a radius $r=r_{0}$ must fulfill following boundary conditions:

- for a clamped plate displacement and slope vanish at the boundary,

$$
W\left(r_{0}\right)=0, \quad \frac{\partial W\left(r_{0}\right)}{\partial r}=0,
$$

- for a simply supported plate displacement and bending moment vanish at the boundary,

$W\left(r_{0}\right)=0$,

$$
M\left(r_{0}\right)=D\left(\frac{\partial^{2} W}{\partial r^{2}}+\frac{\mu}{r_{0}} \frac{\partial W}{\partial r}\right)_{r=r_{0}}=0,
$$

- for a free plate bending moment and shear force vanish at the boundary,

$$
\begin{aligned}
& M\left(r_{0}\right)=D\left(\frac{\partial^{2} W}{\partial r^{2}}+\frac{\mu}{r_{0}} \frac{\partial W}{\partial r}\right)_{r=r_{0}}=0, \\
& Q\left(r_{0}\right)=D \frac{\partial}{\partial r}\left(\frac{\partial^{2} W}{\partial r^{2}}+\frac{\mu}{r_{0}} \frac{\partial W}{\partial r}\right)_{r=r_{0}}=0 .
\end{aligned}
$$

\subsection{Homogenization of central solid plate}

The central solid plate with a thickness $h_{1}$ is composed of an upper layer with parameters $h_{p}, E_{p}, \rho_{p}$, $\mu_{p}$ and a bottom layer with parameters $h_{m}, E_{m}, \rho_{m}$, $\mu_{m}$. The position of a neutral plane (plane without horizontal strains) is supposed to be in a distance $c$ from the upper plate surface (Fig. 3). By the plate homogenization we will assess equivalent parameters of flexural rigidity $D_{1}$, Young's modulus $E_{1}$ and density $\rho_{1}$. For simplicity we assume that the Poisson's ratios of the piezoelectric and elastic layers have close values $\left(\mu_{p}=0.34\right.$ and $\mu_{m}=0.341$ in a case solved in Sec. 3) and therefore, we define an average value 


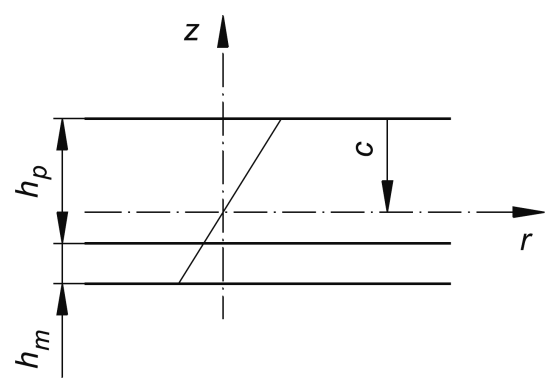

Fig. 3. Central solid plate, position $c$ of neutral plane.

$\mu=\left(\mu_{p}+\mu_{m}\right) / 2$, which holds for the whole unimorph structure.

The position of the neutral plane and the plate equivalent parameters can be found by integrating the radial stress components $\sigma_{r m}$ and $\sigma_{r p}$ towards the thickness direction (AdElman, Stavsky, 1980). We obtain the tension force and bending moment resultants of a symmetrical circular plate from

$$
\begin{aligned}
N_{r} & =\int_{c-h_{p}-h_{m}}^{c-h_{p}} \sigma_{r m} \mathrm{~d} z+\int_{c-h_{p}}^{c} \sigma_{r p} \mathrm{~d} z \\
& =\left(\frac{E_{m}}{1-\mu^{2}} \int_{c-h_{p}-h_{m}}^{c-h_{p}} z \mathrm{~d} z+\frac{E_{p}}{1-\mu^{2}} \int_{c-h_{p}}^{c} z \mathrm{~d} z\right) \frac{\partial^{2} w}{\partial r^{2}}, \\
M_{r} & =\int_{c-h_{p}-h_{m}}^{c-h_{p}} \sigma_{r m} z \mathrm{~d} z+\int_{c-h_{p}}^{c} \sigma_{r p} z \mathrm{~d} z \\
& =\left(\frac{E_{m}}{1-\mu^{2}} \int_{c-h_{p}-h_{m}}^{c} z^{2} \mathrm{~d} z+\frac{E_{p}}{1-\mu^{2}} \int_{c-h_{p}}^{c} z^{2} \mathrm{~d} z\right) \frac{\partial^{2} w}{\partial r^{2}},
\end{aligned}
$$

where radial curvature $\frac{\partial^{2} w}{\partial r^{2}}$ is constant for axisymmetric circular plate.

The tension force resultant $N_{r}$ is zero in the case of a pure bending. After modification of Eq. (12) we can get a position of the neutral plane

$$
\begin{aligned}
c & =\frac{E_{m}\left(1-\mu^{2}\right)\left(h_{p} h_{m}+h_{m}^{2} / 2\right)+E_{p}\left(1-\mu^{2}\right) h_{p}^{2} / 2}{E_{m}\left(1-\mu^{2}\right) h_{m}+E_{p}\left(1-\mu^{2}\right) h_{p}} \\
& =\frac{h_{p} h_{m}+h_{m}^{2} / 2+E_{e} h_{p}^{2} / 2}{h_{m}+E_{e} h_{p}},
\end{aligned}
$$

where we use a notation

$$
E_{e}=\frac{E_{p}}{E_{m}}
$$

The bending moment of homogenous plate of a thickness $h_{1}=h_{p}+h_{m}$ is given by

$$
M_{r}=\frac{E_{1}\left(h_{p}+h_{m}\right)^{3}}{12\left(1-\mu^{2}\right)} \frac{\partial^{2} w}{\partial r^{2}}=D_{1} \frac{\partial^{2} w}{\partial r^{2}} .
$$

Comparing Eq. (16) with (13) and substituting for $c$ from Eq. (14) we can get the equivalent Young's modulus

$E_{1}=E_{m} \frac{h_{m}^{4}+E_{e}\left(4 h_{p}^{3} h_{m}+6 h_{p}^{2} h_{m}^{2}+4 h_{p} h_{m}^{3}\right)+E_{e}^{2} h_{p}^{4}}{\left(h_{m}+E_{e} h_{p}\right)\left(h_{p}+h_{m}\right)^{3}}$

and equivalent flexural rigidity

$$
\begin{aligned}
& D_{1}=\frac{E_{1}\left(h_{p}+h_{m}\right)^{3}}{12\left(1-\mu^{2}\right)}=\frac{E_{m}}{12\left(1-\mu^{2}\right)} \\
& \cdot\left(\frac{h_{m}^{4}+E_{e}\left(4 h_{p}^{3} h_{m}+6 h_{p}^{2} h_{m}^{2}+4 h_{p} h_{m}^{3}\right)+E_{e}^{2} h_{p}^{4}}{h_{m}+E_{e} h_{p}}\right) .
\end{aligned}
$$

To fulfill the condition of the original and equivalent plate equal density per unit area we can get

$$
\rho_{1}=\frac{h_{p} \rho_{p}+h_{m} \rho_{m}}{h_{p}+h_{m}} .
$$

Equations (14) and (17) have to be modified, if the piezoelectric and elastic layers are connected by a very thin layer $\left(h_{a}=0-10 \mu \mathrm{m}\right)$ of an adhesive with Young's modulus, e.g. $E_{a}=30 \mathrm{GPa}$. The influence of this layer on the above mentioned values of the neutral plane position $c$ and on the equivalent Young's modulus $E_{1}$ of the unimorph APC 20-1075 with parameters defined in Sec. 3 is evident from the Table 1.

\subsection{Natural vibration of unimorph plate}

The natural vibration is described by the equation of motion (9) with a general solution $W(r)=V_{1}(r)+$ $V_{2}(r)$, see Eq. (10). From the previous analysis we have following relations for the central equivalent solid plate

$$
\begin{aligned}
D_{1}= & \frac{E_{1}\left(h_{p}+h_{m}\right)^{3}}{12\left(1-\mu^{2}\right)}, \\
\lambda_{1}^{4}= & \frac{\Omega^{2} \rho_{1}\left(h_{p}+h_{m}\right)}{D_{1}}, \\
W_{1}(r)= & A_{1} J_{0}\left(\lambda_{1} r\right)+C_{1} I_{0}\left(\lambda_{1} r\right), \\
\frac{\partial W_{1}(r)}{\partial r}= & -\lambda_{1}\left[A_{1} J_{1}\left(\lambda_{1} r\right)-C_{1} I_{1}\left(\lambda_{1} r\right)\right], \\
M_{1}(r)= & D_{1} A_{1} \lambda_{1}^{2}\left[-J_{0}\left(\lambda_{1} r\right)+\frac{1-\mu}{\lambda_{1} r} J_{1}\left(\lambda_{1} r\right)\right] \\
& +D_{1} C_{1} \lambda_{1}^{2}\left[I_{0}\left(\lambda_{1} r\right)-\frac{1-\mu}{\lambda_{1} r} I_{1}\left(\lambda_{1} r\right)\right], \\
Q_{1}(r)= & D_{1} A_{1} \lambda_{1}^{3} J_{1}\left(\lambda_{1} r\right)+D_{1} C_{1} \lambda_{1}^{3} I_{1}\left(\lambda_{1} r\right)
\end{aligned}
$$


and for the outer annular plate

$$
\begin{aligned}
D_{2}= & \frac{E_{m} h_{m}^{3}}{12\left(1-\mu^{2}\right)}, \\
\lambda_{2}^{4}= & \frac{\Omega^{2} \rho_{2} h_{m}}{D_{2}}, \\
\rho_{2}= & \rho_{m}, \\
W_{2}(r)= & A_{2} J_{0}\left(\lambda_{2} r\right)+B_{2} Y_{0}\left(\lambda_{2} r\right) \\
& +C_{2} I_{0}\left(\lambda_{2} r\right)+D_{2}^{*} K_{0}\left(\lambda_{2} r\right), \\
\frac{\partial W_{2}(r)}{\partial r}= & -\lambda_{2}\left[A_{2} J_{1}\left(\lambda_{2} r\right)+B_{2} Y_{1}\left(\lambda_{2} r\right)\right. \\
& \left.-C_{2} I_{1}\left(\lambda_{2} r\right)+D_{2}^{*} K_{1}\left(\lambda_{2} r\right)\right] \\
M_{2}(r)= & D_{2} A_{2} \lambda_{2}^{2}\left[-J_{0}\left(\lambda_{2} r\right)+\frac{1-\mu}{\lambda_{2} r} J_{1}\left(\lambda_{2} r\right)\right] \\
& +D_{2} B_{2} \lambda_{2}^{2}\left[-Y_{0}\left(\lambda_{2} r\right)+\frac{1-\mu}{\lambda_{2} r} Y_{1}\left(\lambda_{2} r\right)\right] \\
& +D_{2} C_{2} \lambda_{2}^{3} I_{1}\left(\lambda_{2} r\right)-D_{2} D_{2}^{*} \lambda_{2}^{3} K_{1}\left(\lambda_{2} r\right), \\
& +D_{2} C_{2} \lambda_{2}^{2}\left[I_{0}\left(\lambda_{2} r\right)-\frac{1-\mu}{\lambda_{2} r} I_{1}\left(\lambda_{2} r\right)\right] \\
& +D_{2} D_{2}^{*} \lambda_{2}^{2}\left[K_{0}\left(\lambda_{2} r\right)+\frac{1-\mu}{\lambda_{2} r} K_{1}\left(\lambda_{2} r\right)\right], \\
& D_{2} A_{2} \lambda_{2}^{3} J_{1}\left(\lambda_{2} r\right)+D_{2} B_{2} \lambda_{2}^{3} Y_{1}\left(\lambda_{2} r\right) \\
& \\
& \\
& \\
Q_{2}(r) & \\
& \\
& \\
&
\end{aligned}
$$

where $J_{1}, Y_{1}$ are Bessel's functions of the first and second kind and first order and $I_{1}, K_{1}$ are modified Bessel's functions of the first and second kind and first order.

The solution of natural vibrations must fulfill following boundary conditions:

1) the condition of displacement, slope, bending moment and shear force continuity at the boundary of central and outer plate for $r=r_{1}=d_{p} / 2$, i.e.

$$
\begin{aligned}
W_{1}\left(r_{1}\right) & =W_{2}\left(r_{1}\right), & \frac{\partial W_{1}\left(r_{1}\right)}{\partial r} & =\frac{\partial W_{2}\left(r_{1}\right)}{\partial r}, \\
M_{1}\left(r_{1}\right) & =M_{2}\left(r_{1}\right), & Q_{1}\left(r_{1}\right) & =Q_{2}\left(r_{1}\right),
\end{aligned}
$$

2) the mounting conditions on the outer circumference for $r=r_{2}=d_{m} / 2$ (see Eq. (11))

- for a clamped plate

$$
W_{2}\left(r_{2}\right)=0, \quad \frac{\mathrm{d} W_{2}\left(r_{2}\right)}{\mathrm{d} r}=0,
$$

- for a simply supported plate

$$
W_{2}\left(r_{2}\right)=0, \quad M_{2}\left(r_{2}\right)=0,
$$

- or for a free plate

$$
M_{2}\left(r_{2}\right)=0, \quad Q_{2}\left(r_{2}\right)=0 .
$$

Six boundary conditions Eqs. (22) and (23) contain six unknown constants $A_{1}, C_{1}, A_{2}, B_{2}, C_{2}, D_{2}^{*}$. Frequency parameters $\lambda_{1}, \lambda_{2}$ are defined by the flexural stiffnesses $D_{1}, D_{2}$, the material densities $\rho_{1}, \rho_{2}$ and also by unknown resonant frequency $\Omega$ common for both plates. Set of linear Eqs. (22) and (23) can be expressed in a matrix form

$$
\mathbf{H} \mathbf{X}=0 \text {, }
$$

where $\mathbf{H}$ is a coefficient matrix

$$
\mathbf{H}=\left[\begin{array}{cccccc}
a_{11} & a_{12} & a_{13} & a_{14} & a_{15} & a_{16} \\
a_{21} & a_{22} & a_{23} & a_{24} & a_{25} & a_{26} \\
a_{31} & a_{32} & a_{33} & a_{34} & a_{35} & a_{36} \\
a_{41} & a_{42} & a_{43} & a_{44} & a_{45} & a_{46} \\
0 & 0 & a_{53} & a_{54} & a_{55} & a_{56} \\
0 & 0 & a_{63} & a_{64} & a_{65} & a_{66}
\end{array}\right]
$$

and $\mathbf{X}$ is a vector of unknown constants

$$
\mathbf{X}=\left[A_{1}, C_{1}, A_{2}, B_{2}, C_{2}, D_{2}^{*}\right]^{\mathrm{T}} .
$$

Elements in first four rows of the matrix $\mathbf{H}$ are related to the boundary conditions on the plates connection $r=r_{1}$ and have a form

$$
\begin{aligned}
& a_{11}=-J_{0}\left(\lambda_{1} r_{1}\right), \\
& a_{12}=-I_{0}\left(\lambda_{1} r_{1}\right), \\
& a_{13}=J_{0}\left(\lambda_{2} r_{1}\right) \text {, } \\
& a_{14}=Y_{0}\left(\lambda_{2} r_{1}\right), \\
& a_{15}=I_{0}\left(\lambda_{2} r_{1}\right) \\
& a_{16}=K_{0}\left(\lambda_{2} r_{1}\right) \text {, } \\
& a_{21}=\lambda_{1} J_{1}\left(\lambda_{1} r_{1}\right), \\
& a_{22}=-\lambda_{1} I_{1}\left(\lambda_{1} r_{1}\right), \\
& a_{23}=-\lambda_{2} J_{1}\left(\lambda_{2} r_{1}\right) \text {, } \\
& a_{24}=-\lambda_{2} Y_{1}\left(\lambda_{2} r_{1}\right), \\
& a_{25}=\lambda_{2} I_{1}\left(\lambda_{2} r_{1}\right), \\
& a_{26}=-\lambda_{2} K_{1}\left(\lambda_{2} r_{1}\right), \\
& a_{31}=D_{1} \lambda_{1}^{2}\left[J_{0}\left(\lambda_{1} r_{1}\right)-\frac{1-\mu}{\lambda_{1} r_{1}} J_{1}\left(\lambda_{1} r_{1}\right)\right], \\
& a_{32}=D_{1} \lambda_{1}^{2}\left[-I_{0}\left(\lambda_{1} r_{1}\right)+\frac{1-\mu}{\lambda_{1} r_{1}} I_{1}\left(\lambda_{1} r_{1}\right)\right], \\
& a_{33}=D_{2} \lambda_{2}^{2}\left[-J_{0}\left(\lambda_{2} r_{1}\right)+\frac{1-\mu}{\lambda_{2} r_{1}} J_{1}\left(\lambda_{2} r_{1}\right)\right] \text {, } \\
& a_{34}=D_{2} \lambda_{2}^{2}\left[-Y_{0}\left(\lambda_{2} r_{1}\right)+\frac{1-\mu}{\lambda_{2} r_{1}} Y_{1}\left(\lambda_{2} r_{1}\right)\right] \text {, } \\
& a_{35}=D_{2} \lambda_{2}^{2}\left[I_{0}\left(\lambda_{2} r_{1}\right)-\frac{1-\mu}{\lambda_{2} r_{1}} I_{1}\left(\lambda_{2} r_{1}\right)\right] \text {, } \\
& a_{36}=D_{2} \lambda_{2}^{2}\left[K_{0}\left(\lambda_{2} r_{1}\right)+\frac{1-\mu}{\lambda_{2} r_{1}} K_{1}\left(\lambda_{2} r_{1}\right)\right] \text {, } \\
& a_{41}=-D_{1} \lambda_{1}^{3} J_{1}\left(\lambda_{1} r_{1}\right), \quad a_{42}=-D_{1} \lambda_{1}^{3} I_{1}\left(\lambda_{1} r_{1}\right), \\
& a_{43}=D_{2} \lambda_{2}^{3} J_{1}\left(\lambda_{2} r_{1}\right), \quad a_{44}=D_{2} \lambda_{2}^{3} Y_{1}\left(\lambda_{2} r_{1}\right), \\
& a_{45}=D_{2} \lambda_{2}^{3} I_{1}\left(\lambda_{2} r_{1}\right), \quad a_{46}=-D_{2} \lambda_{2}^{3} K_{1}\left(\lambda_{2} r_{1}\right) \text {. }
\end{aligned}
$$


Last two rows are related to boundary conditions on the outer circumference $r=r_{2}$ and have a form

- for the clamped plate

$$
\begin{array}{ll}
a_{53}=J_{0}\left(\lambda_{2} r_{2}\right), & a_{54}=Y_{0}\left(\lambda_{2} r_{2}\right), \\
a_{55}=I_{0}\left(\lambda_{2} r_{2}\right), & a_{56}=K_{0}\left(\lambda_{2} r_{2}\right), \\
a_{63}=-J_{1}\left(\lambda_{2} r_{2}\right), & a_{64}=-Y_{1}\left(\lambda_{2} r_{2}\right), \\
a_{65}=I_{1}\left(\lambda_{2} r_{2}\right), & a_{66}=-K_{1}\left(\lambda_{2} r_{2}\right),
\end{array}
$$

- for the simply supported plate

$$
\begin{aligned}
& a_{53}=J_{0}\left(\lambda_{2} r_{2}\right), \quad a_{54}=Y_{0}\left(\lambda_{2} r_{2}\right), \\
& a_{55}=I_{0}\left(\lambda_{2} r_{2}\right), \quad a_{56}=K_{0}\left(\lambda_{2} r_{2}\right), \\
& a_{63}=D_{2} \lambda_{2}^{2}\left[-J_{0}\left(\lambda_{2} r_{2}\right)+\frac{1-\mu}{\lambda_{2} r_{2}} J_{1}\left(\lambda_{2} r_{2}\right)\right], \\
& a_{64}=D_{2} \lambda_{2}^{2}\left[-Y_{0}\left(\lambda_{2} r_{2}\right)+\frac{1-\mu}{\lambda_{2} r_{2}} Y_{1}\left(\lambda_{2} r_{2}\right)\right], \\
& a_{65}=D_{2} \lambda_{2}^{2}\left[I_{0}\left(\lambda_{2} r_{2}\right)-\frac{1-\mu}{\lambda_{2} r_{2}} I_{1}\left(\lambda_{2} r_{2}\right)\right], \\
& a_{66}=D_{2} \lambda_{2}^{2}\left[K_{0}\left(\lambda_{2} r_{2}\right)+\frac{1-\mu}{\lambda_{2} r_{2}} K_{1}\left(\lambda_{2} r_{2}\right)\right],
\end{aligned}
$$

- or for the free plate

$$
\begin{aligned}
& a_{53}=D_{2} \lambda_{2}^{3} J_{1}\left(\lambda_{2} r_{2}\right), \\
& a_{54}=D_{2} \lambda_{2}^{3} Y_{1}\left(\lambda_{2} r_{2}\right), \\
& a_{55}=D_{2} \lambda_{2}^{3} I_{1}\left(\lambda_{2} r_{2}\right), \\
& a_{56}=-D_{2} \lambda_{2}^{3} K_{1}\left(\lambda_{2} r_{2}\right), \\
& a_{63}=D_{2} \lambda_{2}^{2}\left[-J_{0}\left(\lambda_{2} r_{2}\right)+\frac{1-\mu}{\lambda_{2} r_{2}} J_{1}\left(\lambda_{2} r_{2}\right)\right], \\
& a_{64}=D_{2} \lambda_{2}^{2}\left[-Y_{0}\left(\lambda_{2} r_{2}\right)+\frac{1-\mu}{\lambda_{2} r_{2}} Y_{1}\left(\lambda_{2} r_{2}\right)\right], \\
& a_{65}=D_{2} \lambda_{2}^{2}\left[I_{0}\left(\lambda_{2} r_{2}\right)-\frac{1-\mu}{\lambda_{2} r_{2}} I_{1}\left(\lambda_{2} r_{2}\right)\right], \\
& a_{66}=D_{2} \lambda_{2}^{2}\left[K_{0}\left(\lambda_{2} r_{2}\right)+\frac{1-\mu}{\lambda_{2} r_{2}} K_{1}\left(\lambda_{2} r_{2}\right)\right] .
\end{aligned}
$$

It is convenient to substitute the two frequency parameters $\lambda_{1}, \lambda_{2}$ with one value by introducing a ratio $\alpha=\lambda_{1} / \lambda_{2}$, where

$$
\alpha^{4}=\frac{\lambda_{1}^{4}}{\lambda_{2}^{4}}=\frac{D_{2} \rho_{1} h_{1}}{D_{1} \rho_{2} h_{2}} .
$$

A nontrivial solution of Eq. (24) exists if the determinant of the matrix $\mathbf{H}$ is equal zero, which yields the frequency parameter $\lambda_{2}=\lambda_{1} / \alpha$. Substituting these values into Eq. (21) for $\lambda_{2}^{4}$ we can get angular resonant frequencies $\Omega$ of the unimorph.
The mode shapes for a given value of frequency $\Omega$ can be calculated using Eqs. (20) and (21) for $W_{1}(r)$ and $W_{2}(r)$. The amplitude ratio

$$
\begin{aligned}
A_{1}: C_{1}: A_{2}: B_{2}: C_{2}: D_{2}^{*} \\
\quad=H_{1}:-H_{2}: H_{3}:-H_{4}: H_{5}:-H_{6}
\end{aligned}
$$

could be obtained from the ratio of subdeterminants $H_{k}$, which arise from the matrix $\mathbf{H}$, e.g. by omitting the first row and the $k$-th column.

\section{Calculation results and comparison with measurement}

The computational method was applied to a spectral analysis of APC 20-1075 unimorph sample composed of APC 855 piezoceramic and brass layers with following material parameters: $E_{p}=59 \mathrm{GPa}, \mu_{p}=$ $0.34, \rho_{p}=7600 \mathrm{~kg} \mathrm{~m}^{-3}$ and $E_{m}=110 \mathrm{GPa}, \mu_{m}=$ 0.341 and $\rho_{m}=8500 \mathrm{~kg} \mathrm{~m}^{-3}$. The unimorph dimensions (see Fig. 1) were $\varnothing d_{m}=48 \mathrm{~mm}, \varnothing d_{p}=25 \mathrm{~mm}$, $h_{m}=0.15 \mathrm{~mm}$ and $h_{p}=0.12 \mathrm{~mm}$. For calculation an average value of the Poisson's ratio $\mu=0.3405$ was used. Theoretical resonant frequencies and corresponding shapes of three lowest modes were calculated by the solution of Eq. (24) for boundary conditions of clamped, simply supported and free unimorph circumference. The calculated mode shapes are depicted in Figs. 4-6.

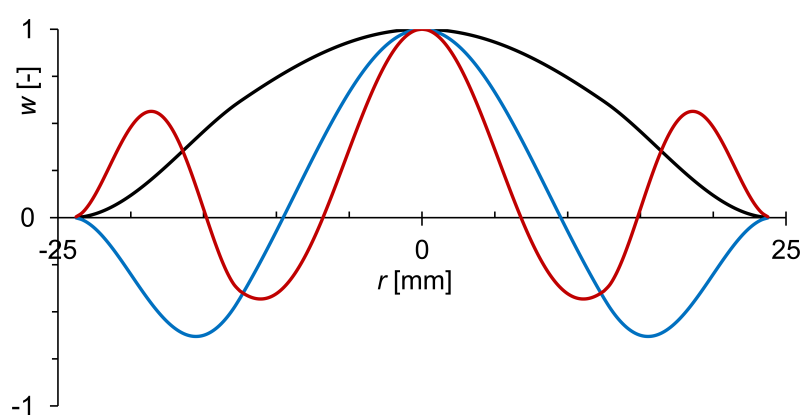

Fig. 4. Three lowest mode shapes for the clamped circumference.

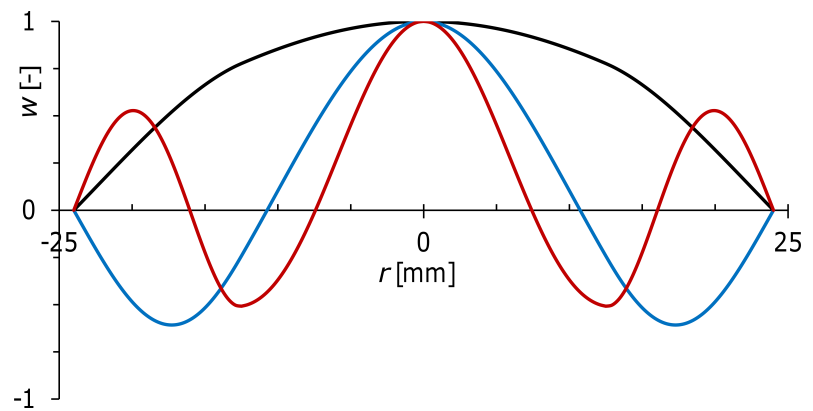

Fig. 5. Three lowest mode shapes for the simply supported circumference. 


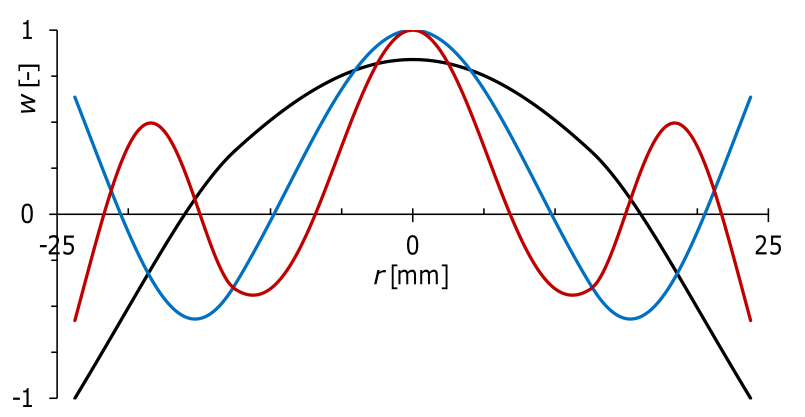

Fig. 6. Three lowest mode shapes for the free circumference.

The resonant frequencies of the unimorph sample were found experimentally by means of the vibration velocity measurement. The sample was clamped by screws between two plastic flanges and mounted in a large wooden plate. This mounting type corresponds to a rigid clamping at the circumference $d_{m}$. The vibration velocity of the unimorph center was measured at a distance of $0.9 \mathrm{~m}$ above the unimorph plane in the unimorph axis by a vibration analyzer Polytec PDV100. The sample was excited by a swept harmonic signal with amplitude $1 \mathrm{~V}$ in the frequency range from $200 \mathrm{~Hz}$ to $5 \mathrm{kHz}$. The recorded signals were processed in a frequency domain using a measurement system Brüel \& Kjaer PULSE. The experimental setup is depicted in Fig. 7. To suppress the noise influence in the measured data all measurements were repeated many times and the frequency spectra were averaged. The resulting velocity frequency spectrum shown in Fig. 8 is processed in the form of a frequency response function, i.e. as a normalized velocity spectrum divided by a spectrum of excitation voltage. The resonant frequencies corresponding to vibration velocity maxima are clearly evident.

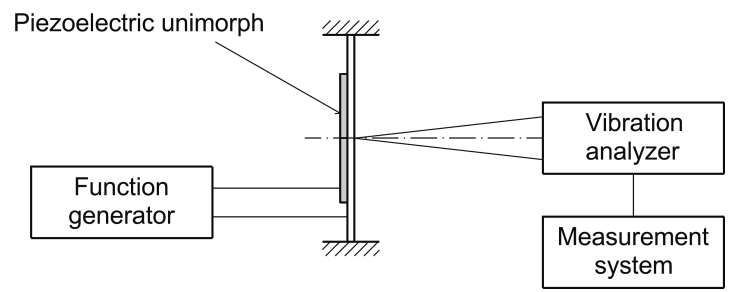

Fig. 7. Measurement system used for experimental estimation of unimorph resonant frequencies.

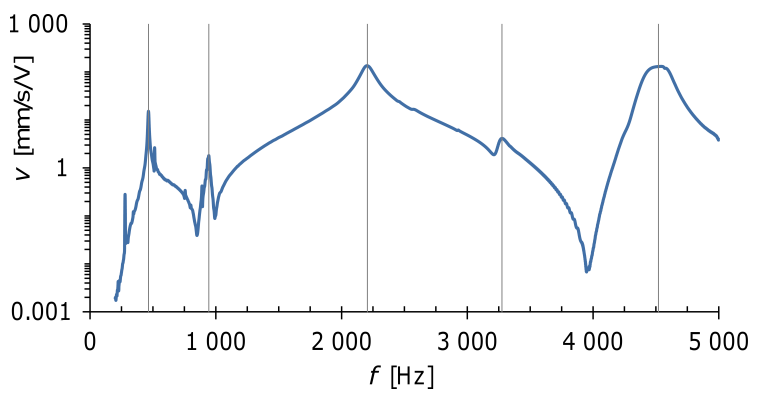

Fig. 8. Frequency spectrum of unimorph center vibration velocity.
The comparison of the calculated resonant frequencies with the measured values is presented in Table 2 . The mounting at unimorph circumference has a finite stiffness and measured values should be observed between the theoretical frequencies for the clamped and simply supported boundary conditions. Despite the simplifications applied in the calculation model the measured values are close to the theoretical ones for the clamped boundary and agreement of the measured and calculated frequencies is convenient for practical purposes.

Table 2. Comparison of theoretical and measured resonant frequencies $[\mathrm{Hz}]$ for various boundary conditions on unimorph outer circumference.

\begin{tabular}{|c|c|c|c|c|}
\hline $\begin{array}{c}\text { Mode } \\
\text { number }\end{array}$ & $\begin{array}{c}\text { Model } \\
\text { clamped }\end{array}$ & $\begin{array}{c}\text { Model } \\
\text { simply } \\
\text { supported }\end{array}$ & $\begin{array}{c}\text { Model } \\
\text { free }\end{array}$ & Measurement \\
\hline 1 & 508 & 270 & 571 & 464 \\
\hline & & & & 944 \\
\hline 2 & 2177 & 1660 & 2108 & 2205 \\
\hline & & & & 3276 \\
\hline 3 & 5088 & 4142 & 5002 & 4520 \\
\hline
\end{tabular}

Additional modes occur in the measured frequency spectrum in Fig. 8 (i.e. frequencies 944 and $3276 \mathrm{~Hz}$ in Table 2) having lower amplitude response than the "pure" modes. This is apparently an effect of small asymmetry in the unimorph structure resulting from imperfect mounting, misalignment of the piezoceramic and metal layers etc., which causes the excitation of non-axisymmetric vibration modes having also nodal lines in addition to nodal diameters. The solution for these modes can be included in the calculation model by introducing $n>0$ in Eq. (8).

\section{Influence of thickness tolerance on the resonant frequency}

As follows from Eq. (6), the resonant frequency $\Omega$ is proportional to the thickness of the unimorph layers. Low-cost commercially available piezoelectric unimorphs are produced with a thickness tolerance of tens of percent of the nominal value. Such a variation has a significant influence on the resonant frequency. The analytical model derived in this paper was used to investigate the effect of the thickness tolerance on the APC 20-1075 unimorph resonant frequencies. This analysis was necessary in a design process of an acoustic device comprising this unimorph as a main component.

The aim of the analysis was an estimation of the resonant frequency change in case the layer thickness varies between $90 \%$ and $110 \%$ of its nominal value. The calculation was performed separately for the piezoelectric layer $h_{p}$ and the elastic layer $h_{m}$. The nominal geo- 
metrical and material parameters used for the analysis are defined in Sec. 3. The dependence of the resonant frequency on the layer thickness was obtained by successive solution of Eq. (24), where the $h_{p}$ or $h_{m}$ values were varied with a step of $0.1 \%$ of its nominal value. The resulting resonant frequencies are also expressed as percentual changes of their nominal values.

Dependence of the frequency relative change on the layer thickness is shown in Fig. 9 for unimorph clamped at the circumference. These results were obtained by varying only one layer thickness, while the other layer thickness being constant. In studied dimension range the resonant frequency is virtually linearly dependent. This dependency is higher for the elastic layer changes. While the frequency relative change is about $2.1 \%$ for the 2 nd mode at the piezoelectric layer limit $+10 \%$, the change is up to $8.9 \%$ for the 1 st mode at the same elastic layer limit. The frequency dependence on both thickness variations is depicted in Fig. 10 for the 1st
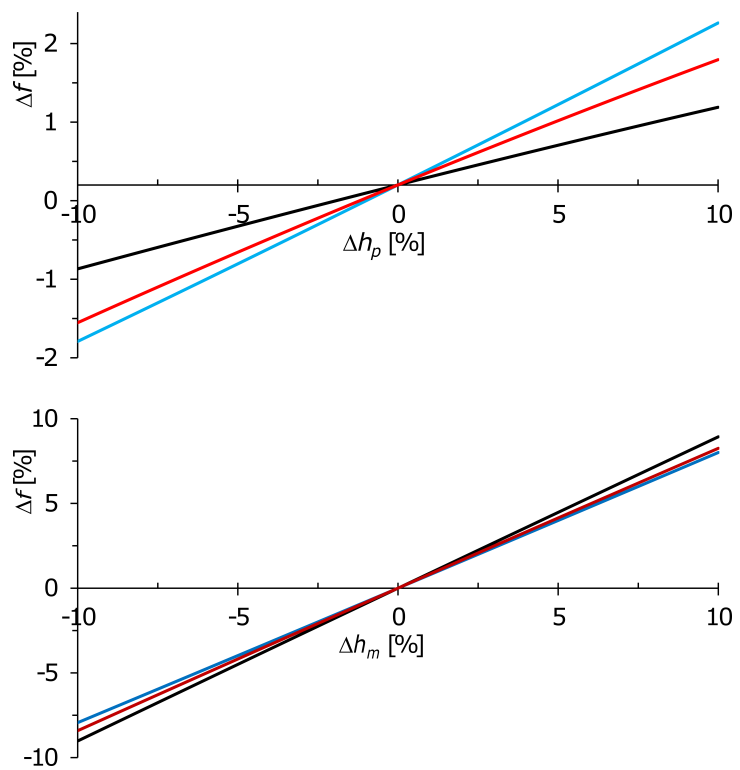

Fig. 9. Dependence of the relative change of frequency on the relative change of piezoelectric (top) and elastic layer thickness (bottom), clamped circumference, black 1st mode, blue - 2nd mode, red - 3rd mode.

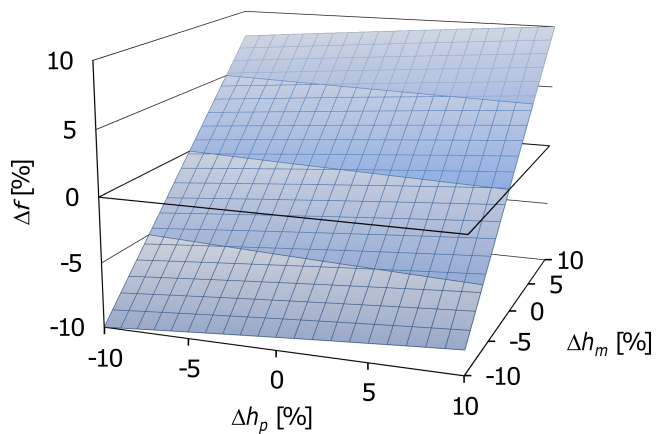

Fig. 10. Dependence of the relative change of frequency on the relative change of layer components thickness (clamped circumference, 1st mode). mode. The change in total thickness $h_{p}+h_{m}$ of $10 \%$ is the cause of the frequency relative change of approximately $9.9 \%$.

\section{Conclusion}

The analytical model based on the Kirchhoff's laminated plate theory was developed to describe the natural vibrations of piezoelectric unimorph with different diameters of the piezoelectric and elastic layers. The derived system of equations allows the calculation of the resonant frequencies and the mode shapes for clamped, simply supported and free boundary conditions on the unimorph outer circumference. The theoretical resonant frequencies were compared with the vibration velocity measurement of the clamped unimorph sample. The calculated frequencies agree well with the experimental values and the computational model is applicable for initial design and optimization purposes with respect to the non-linear properties of piezoelectric ceramics and a high variability of its material constants.

The additional vibration modes which are not described by the calculation model were identified experimentally in the frequency spectrum. They have apparently non-axisymmetric shape and arise from small geometrical and material properties unbalance in the unimorph structure. These modes can be easily included in the model by introducing a solution function having nodal lines and nodal diameters.

\section{Acknowledgment}

This work was supported by the Czech Ministry of Education, project LO1213. The authors would like to thank Professor Jiří Erhart from Technical University of Liberec for his valuable advice and providing unimorph samples for experimental work.

\section{References}

1. Adelman N.T., Stavsky Y. (1980), Flexuralextensional behavior of composite piezoelectric circular plates, The Journal of the Acoustical Society of America, 67, 3, 819-822.

2. Deshrande M., Saggere L. (2007), An analytical model and working equations for static deflections of a circular multi-layered diaphragm-type piezoelectric actuator, Sensors and Actuators A: Physical, 136, 2, 673-689.

3. Dobrucki A.B., Pruchnicki P. (1997), Theory of piezoelectric axisymmetric bimorph, Sensors and Actuators A: Physical, 58, 3, 203-212.

4. Dong S., Uchino K., Li L., Viehland D. (2007), Analytical solutions for the transverse deflection of a piezoelectric circular axisymmetric unimorph actuator, IEEE Transactions on Ultrasonics, Ferroelectrics, and Frequency Control, 54, 6, 1240-1249. 
5. Gazis D.C., Minduin R.D. (1960), Extensional vibrations and waves in a circular disk and a semi-infinite plate, Journal of Applied Mechanics, 27, 3, 541-547.

6. Leissa A.W. (1969), Vibration of plates, NASA, SP160, Washington D.C.

7. Li S., CHen S. (2003), Analytical analysis of a circular PZT actuator for valveless micropumps, Sensors and Actuators A: Physical, 104, 2, 151-161.

8. Li X., Sнin W.Y., Aksay I.A., Shin W.H. (1999), Electromechanical behavior of PZT-brass unimorphs, Journal of the American Ceramic Society, 82, 7, 17331740 .

9. Liu C., Cui T., Zhou Z. (2003), Modal analysis of a unimorph piezoelectrical transducer, Microsystem Technologies, 9, 6-7, 474-479.

10. Mo C., Wright R., Slaughter W.S., Clark W.W. (2006), Behaviour of a unimorph circular piezoelectric actuator, Smart Materials and Structures, 15, 4, 10941102 .
11. Mo C., Radziemski L.J., Clark W.W. (2010), Analysis of piezoelectric circular diaphragm energy harvesters for use in a pressure fluctuating system, Smart Materials and Structures, 19, 2, 025016.

12. Papila M., Sheplak M., Cattafesta L.N. (2008), Optimization of clamped circular piezoelectric composite actuators, Sensors and Actuators A: Physical, 147, $1,310-323$.

13. Prasad S.A.N., Gallas Q., Horowitz S., Homeijer B., Sankar B.V., Cattafesta L.N., ShePLAK M. (2006), Analytical electroacoustic model of a piezoelectric composite circular plate, AIAA Journal, 44, 10, 2311-2318.

14. Wang Q., Quek S.T., Sun C. T., Liu X. (2001), Analysis of piezoelectric coupled circular plate, Smart Materials and Structures, 10, 2, 229-239.

15. Yang J., Zhou H., Dong S. (2006), Analysis of plate piezoelectric unimorphs, IEEE Transactions on Ultrasonics, Ferroelectrics, and Frequency Control, 53, 2, $456-462$. 